SPICA Workshop, 04016 (2009)

DOI:10.1051/spica/200904016

(C) Owned by the authors, published by EDP Sciences, 2009

This is an Open Access article distributed under the terms of the Creative Commons Attribution-Noncommercial License, which permits unrestricted use, distribution, and reproduction in any noncommercial medium, provided the original work is properly cited.

\title{
SEARCHING FOR HEAVILY OBSCURED AGN AT HIGH REDSHIFT WITH THE SAFARI-SPICA SPECTRO-PHOTOMETER
}

\author{
F. Pozzi ${ }^{1}$, C. Gruppioni ${ }^{2}$, C. Vignali ${ }^{1}$, A.Comastri ${ }^{2}$, L. Spinoglio ${ }^{3}$ and M.Magliocchetti ${ }^{3}$ \\ ${ }^{1}$ Dipartimento di Astronomia, Bologna, Italy \\ ${ }^{2}$ Istituto Nazionale di Astronomia, Osservatorio Astronomico di Bologna, Via Ranzani 1, I-40127 Bologna, Italy \\ ${ }^{3}$ Istituto Nazionale di Astronomia, IFSI, Via del Fosso del Cavaliere 100, I-00133 Roma, Italy
}

\begin{abstract}
Heavily obscured and Compton-thick Active Galactic Nuclei (AGN) may remain undetected even in the deepest $\mathrm{X}$-ray and optical surveys. In the last few years, thanks to Spitzer observations, it has been realized that mid-IR (MIR) observations can provide a viable and, in some cases, unique alternative to X-ray and optical searches for the most obscured AGN. The Spitzer spectrometer IRS has identified some of these sources up to $z \sim 2.5$ (due to the spectral coverage), and, by characterizing the MIR spectrum, has allowed to disentangle the different contribution of accretion and star-formation processes to the observed MIR emission. The spectro-photometry mode of $S A F A R I$, on board of $S P I C A$, will extend the IRS study to higher redshift $(>2.5-3)$, characterizing the restframe MIR spectrum of dust-obscured galaxies and AGNs. This will allow us to witness the dust-enshrouded blackhole/galaxy formation epoch.
\end{abstract}

Key words: Cosmology: observations - Galaxies: active Infrared: galaxies - Missions: SPICA

\section{INTRODUCTION}

Recent evidences that almost all galaxies contain a super massive black hole (SMBHs) in its center, whose mass is proportional to that of its host (Magorrian et al. 1998), suggest that the growth of galaxies and SMBHs are intimately connected. These observational results have been supported by hydrodynamical simulations (e.g. Di Matteo et al. 2005; Hopkins et al. 2006; Menci et al. 2008) of galaxy evolution, where AGNs provide feedback between SMBHs and host galaxies (Menci et al. 2008 and references with in). One of the most important distinguishing features of the current evolutionary scheme of galaxy and black holes co-evolution, is presented by the early phases, expected to be obscured by large amounts of cold gas and dust (Fabian et al. 1998). In this heavily obscured phase, a strong starburst and an obscured AGN would probably coexist and give significant contribution to the far-IR/sub$\mathrm{mm}$ band (e.g. Page et al. 2004; Vignali et al. 2009). Therefore, a complete census of highly obscured AGN, although challenging to be obtained, is of fundamental importance to understand galaxy and SMBHs formation and evolution.

Observations in the hard X-ray band have been proven to be an efficient tool to select unobscured and moderately obscured AGN (or Compton-thin, for which the absorbing column density $N_{H}$ is $<10^{24} \mathrm{~cm}^{-2}$ ). Anyway, even with the deepest Chandra and XMM-NEWTON observations (e.g. Tozzi et al. 2006) only a handful of the more obscured AGN (or Compton-thick, CT, for for which the absorbing column density $N_{H}$ is $>10^{24} \mathrm{~cm}^{-2}$ ) have been reveal.

On the other hand, some of the energy absorbed by the obscuring material is reprocessed and re-emitted at longer wavelenghts, providing the opportunity to detect the highly obscured AGN via their IR emission (e.g. Pozzi et al. 2007). The strength of the IR-selection method has been shown in the past years by the Spitzer Satellite. In particular, the more efficient way to select highly obscured AGN consists in selecting bright MIR sources (i.e. with AGN luminosities) and faint optical or near-IR emission. Different authors (e.g. Houck et al. 2005; Weedman et al. 2007; Polletta et al. 2008), have shown how the majority of a large sample (some tens) of bright $24 \mu \mathrm{m}$ sources $(S(24 \mu \mathrm{m})>0.7 \mathrm{mJy})$ with faint optical counterparts are indeed AGN dominated. In the above cited studies, the IRS spectrometer is fundamental to detect the strong silicate absorption feature at $9.7 \mu \mathrm{m}$, a strong indicator of obscured AGN, coupled with the weak PAH features. The selection criteria via extreme mid-IR to optical colours, to pick heavily obscured AGN, is supported also by independent studies. In Daddi et al. (2007) and Fiore et al. (2008, 2009 ), in fact, the presence of an obscured AGN is done statistically via the stacking on Chandra images sources at the positions of MIR sources selected on the basis of the mid-IR to optical colour.

Here we present how the spectro-photometry mode of the SAFARI instrument, on board on SPICA, will extend the search, and discovers, this class of sources at higher redshifts $(\mathrm{z}>2.5)$. The SAFARI $35-60 \mu \mathrm{m}$ band is the best band to search for AGNs at high- $z$ since the MIR emission (where the AGN could dominate the star-formation emission) move to this band at $z \sim 2-3$. 


\section{A BACKWARD EVOLUTION MODEL FOR IR GALAXIES}

We use the new backward evolution model for galaxy and AGNs in the IR band by Gruppioni \& Pozzi (2009, in preparation) to predict the number counts of obscured AGNs. An estimate of the expected number of sources is necessary to discuss the best strategy to detect them. The assumed model is based on galaxy and AGNs selected in the mid-IR band and is able to accurately reproduce all the observables (source counts, redshift and luminosity distribution) from mid-IR to far-IR. We choose a model developed in the IR rather than a model developed at higher frequencies (e.g. X-ray frequencies) to avoid further assumptions to translates predictions in one band to another one (e.g. Spectral Energy Distributions, SEDs). The model considers four different populations of sources: galaxies (from Sb spiral to extreme starburst), Seyfert 2, Type I AGN and composite sources (AGN + starburst). The relative fractions of the different populations are based on a broad-band SED analysis (Gruppioni et al. 2008) and different evolutionary properties are considered for each population. The model templates used to fit the observed SEDs are taken from the IR library of Polletta et al. (2007). The main feature of the Gruppioni $\&$ Pozzi model is the high percentage ( $\sim 50 \%)$ of mid-IR sources with an AGN dominating in at least one spectral range (mainly in the MIR where the host spectral galaxy has a minimum). This fraction is significantly higher than from optical spectroscopy $(\sim 29 \%)$.

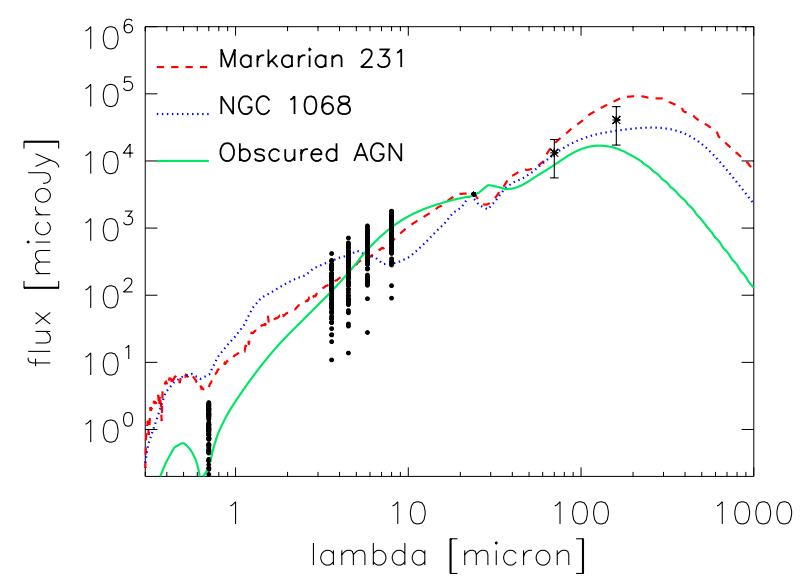

Figure 1. Observed SED for a sample of obscured AGN candidates sources from the SWIRE survey, normalized to $24 \mu \mathrm{m}$. Different obscured $A G N$ at $z=2$ are oplotted to the data points (red dashed curve: Mrk 231; blu dotted curve: NGC 1068; green solid curve from Vignali et al. 2009). The star points at 70 and $160 \mu \mathrm{m}$ are the flux densities obtained by stacking the 70 and 160 Spitzer images.

\subsection{SED OF OBSCURED AGN}

We have considered the composite population, from the model of Gruppioni \& Pozzi (2009, in preparation), as representative of the obscured AGN population. In fact, obscured AGNs at $z \sim 2-3$ should contain both SMBHs and star-formation activity. The template SED used in the model to describe this population is the template SED of Markarian 231 (Mrk 231). Mrk 231 is one of the best known and studied heavily obscured broad absorption-line quasars (BALQSOs), with the AGN dominating the whole observed SED from UV-optical to mid-IR. Different studies show evidences for its far-IR emission being dominated by a strong starburst component (e.g. Fritz et al. 2006). Moreover, the Mrk 231 SED is one of the known templates (together with the model SED reproducing an obscured luminous X-ray selected quasar at $z=2$, see Vignali et al. 2009) that best-fit the observed SEDs of a large sample of obscured AGN candidates selected at $z=1.5-3$. (Gruppioni \& Vignali 2008) in the SWIRE survey (Lonsdale et al. 2004) selected according to the Fiore et al. (2008, 2009) colour technique (see Fig. 1). For these sources, all bright in the MIR $(S(24 \mu \mathrm{m})>2 \mathrm{mJy})$ and with extreme midIR to optical colours $(S(24 \mu m) / S(R)>1250)$, a stacking analysis has been performed on the Spitzer $70 \& 160 \mathrm{im}$ ages, resulting in the detection of 70 and 160 stacked flux at a high level of significance.

\subsection{Model RESUlts}

In Fig. 2 (Left Panel) we show the predicted redshift distributions of the four populations at the nominal flux limit of $0.4 \mathrm{mJy}$, in the SAFARI 35-60 $\mu \mathrm{m}$ band. Considering the composite population (magenta dotted), clearly the number of sources increases at increasing redshift, from being a very rare population in the local Universe up to dominating the counts at $z \sim 2-3$. Moreover, at fainter flux limits, the model predicts a redshift peak for this population at higher $z(z \sim 3$ for $S=0.4 \mathrm{mJy}$ and $z \sim 4$ for $S=0.1$ mJy, Fig. 2, Right panel).

\section{Observational Strategy}

The best strategy for finding and identifying extreme high$z$ obscured AGN is to pre-select candidate objects through an appropriate color selection technique and then to use the spectro-photometry mode of SAFARI at 35-60 $\mu \mathrm{m}$ at low resolution $(R \sim 100)$ to identify them. These observations will resemble those performed by the Spitzer-IRS spectrometer at similar resolution. Anyway, since the $S A$ $F A R I$ spectromether covers a band at longer wavelengths with respect to $\operatorname{IRS}(35-60 \mu \mathrm{m}$ instead of 5-38 $\mu \mathrm{m})$, it will extend to higher $z$ (i.e. $2.6<z<5$ ) the Universe explored by Spitzer, by detecting the $9.7 \mu \mathrm{m}$ strong silicate feature.

A blind spectro-photometric survey is not recommended to detect the proposed population given the low surface density of these sources (see Table 1) and the nominal 

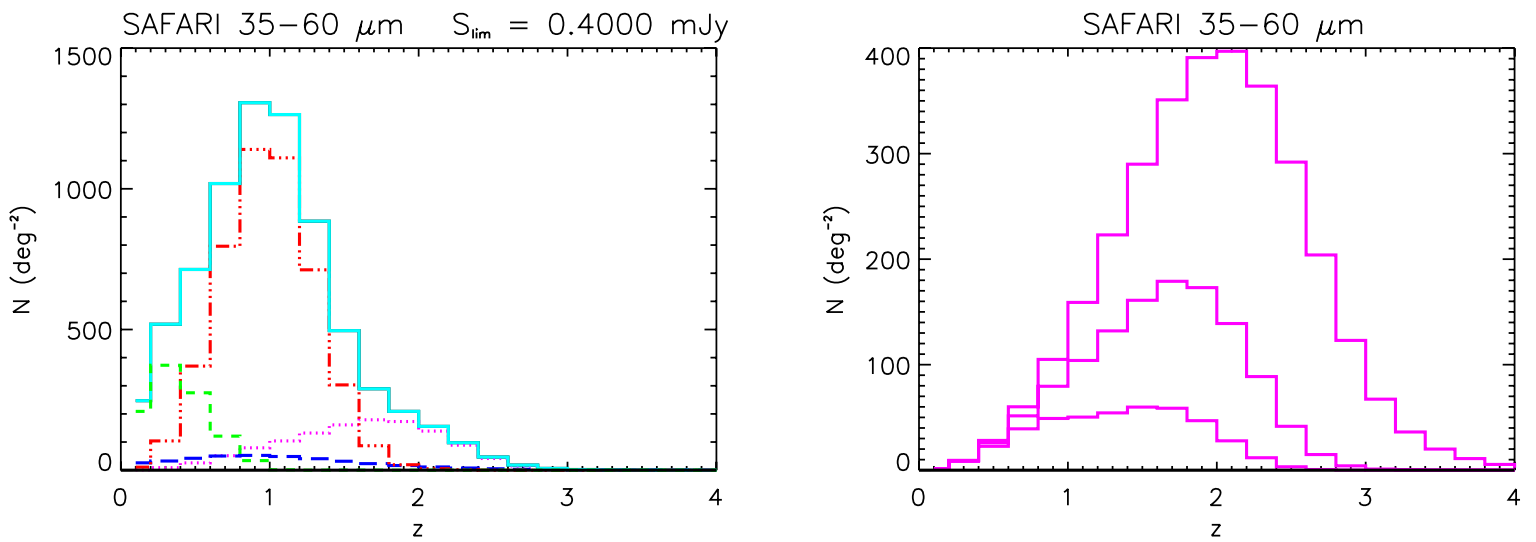

Figure 2. Left: Predicted redshift distribution of the sources at the limiting flux of $0.4 \mathrm{mJy}$ in the SAFARI 35-60 $\mu m$ band, from the new IR backward evolutionary model of Gruppioni \& Pozzi (2009, in preparation). The contribution of the different populations are represented by different colours lines (cyan solid: total; green dashed: galaxies; magenta dotted: composite; red dot-dot-dot-dashed: Seyfert 2; blue long-dashed: AGN1). Right: Predicted redshift distribution of only the composite (starburst

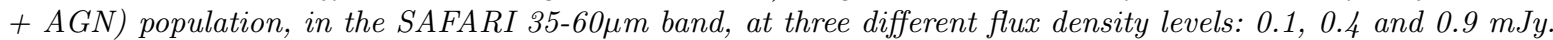

spectro-photometry sensitivity at $35-60 \mu \mathrm{m}(S=0.9 \mathrm{mJy}$ in $1 \mathrm{hr})$. At a nominal flux limit of $0.45 \mathrm{mJy}$, reached with a relatively long integration time $(\sim 4$ hours $)$, in fact only $\sim 120$ candidates are predicted, in a COSMOS-like field $\left(\sim 2 \mathrm{deg}^{2}\right)$, among a total number of $\sim 15000$ MIR sources (see Fig. 1, Left panel). Pre-selection techniques are then mandatory.

We suggest a colour selection technique similar to that proposed by Fiore et al. (2009, see also Dey et al. 2008, Daddi et al. 2007). Via a simple optical to mid-IR color selection, the authors identify highly dust-obscured galaxies (DOGs) with an high percentage of CT AGNs. In particular, at $S(24 \mu \mathrm{m}) / S(R)>1000$, the percentage of CT AGNs is very high $(\sim 90 \%)$ and the typical redshift of the sources is $\sim 2$. The percentage of CT AGN and their typical redshift decreases moving to lower flux ratios (i.e. $\sim 50 \%$ at $30<S(24 \mu m) / S(R)<1000$ with typical redshift of $\sim 1)$. Fiore et al. (2009) estimate the fraction of highly obscured AGNs by stacking Chandra sources selected on the basis of the $S(24 \mu m) / S(R)$ flux ratio, finding a significant signal in the hard X-ray band, with an X-ray flux and hardness ratio strongly suggesting the presence of a highly obscured AGN population. The stacking technique results are tested using Monte Carlo simulations.

The rest-frame spectrum range sampled by the $24 \mu \mathrm{m}$ band, for an object at a typical redshift of 1.5, is sampled by the $S A F A R I 35-60 \mu \mathrm{m}$ band at $z \sim 3$; in a similar way, at $z \sim 3$, the rest-frame sampled by the $R$ band enters the $N I R$ band (e.g. $J$ band). Consequently, a selection using the $S(35-60 \mu m) / S(N I R)$ flux ratio criterion can be used to select a sample of candidate CT AGNs at $z \sim 3$.

In particular, considering a field of $\sim 1 \mathrm{deg}^{2}$, it will be possible to identify $\sim 60$ highly obscured AGN with an integration time of $\sim 240$ hours (not considering the overheads) down to a flux limit of $0.4 \mathrm{mJy}$ in the $35-60 \mu \mathrm{m}$ band. To reduce the observing time, one could consider to reduce the field, reminding that the candidates objects are extreme sources and a large area is necessary to detect them. Observing a GOODS-like field $\left(\sim 150 \operatorname{arcmin}^{2}\right)$, for instance, at the limiting flux of $0.4 \mathrm{mJy}$ too few sources are expected $(<10)$, while moving to lower fluxes (e.g. 0.1 mJy), where a significant number of sources are expected $(\sim 30$, see Table 1$)$ even at $z=3-3.5$, the integration time to detect them would be prohibitive ( $\sim 2000$ hours).

\begin{tabular}{lccc}
$\mathrm{z}$ & \multicolumn{3}{c}{$\mathrm{S}$} \\
\hline & $0.9 \mathrm{mJy}$ & $0.4 \mathrm{mJy}$ & $0.1 \mathrm{mJy}$ \\
\hline $2.0-2.5$ & 39. & 227 & 761 \\
$2.5-3.0$ & 4 & 60. & 619 \\
$3.0-3.5$ & 0. & 1. & 123 \\
\hline
\end{tabular}

Table 1. Predicted number of objects (/deg $\left.{ }^{2}\right)$ of the composite (AGN + starburst) population from the new backward evolutionary model of Gruppioni 85 Pozzi (2009, in preparation) at

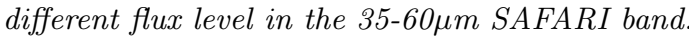

\section{Discussion and Conclusion}

To understand the demography and to characterize the population of highly obscured AGN as a function of redshift is one of the main goal of the present cosmology, since observational and theoretical arguments suggest that the growth of SMBH, residing in AGN, is strictly connected to the growth of the whole galaxy population, and that the first epoch of formation of these structures is highly obscured. While the X-ray band provide one of the best observational window to select unobscured and moderately 
obscured AGN, it misses a significant fraction of the population of highly obscured AGNs.

Previous IR missions (i.e. ISO, Spitzer) have shown how an efficient way to detect and characterize them is from IR-observations, where some of the energy absorbed by the obscuring material is reprocessed and re-emitted.

In this context, the $S A F A R I$ instrument at $35-60 \mu \mathrm{m}$ will be the only future instrument which will cover the mid/far-IR spectral gap between the in-orbit far-IR $H E R$ $S C H E L$ Telescope and the next generation Telescope JWST. Among the observing modes of $S A F A R I$, in this contribution we have highlighted the importance of the $S A$ FARI $35-60 \mu \mathrm{m}$ spectro-photometry mode at low resolution, which will provide an unique means to identify extremely obscured AGN at high-redshift (i.e. $z \sim 2.5-3$ ), by extending to the very distant Universe, the excellent study already performed by the IRS instrument up to $z \sim 2-2.5$.

Since highly obscured AGNs at high- $z$ are extremely rare, we have discussed the best strategy to identify them via mid-IR spectroscopic follow-up of pre-selected targets within a large field. Photometric observations in the $S A$ $F A R I$ bands, combined with data in the NIR, will provide the colours needed to select high- $z$ obscured candidates to be observed and confirmed with $S A F A R I$ spectrophotometric observations. In a field of $\sim 1 \mathrm{deg}^{2}$, we expect to reveal $\sim 60$ sources in $\sim 240$ hours to a flux density of $S(35-60 \mu \mathrm{m})=0.4 \mathrm{mJy}$.

\section{ACKNOWLEDGEMENTS}

This work has benefited for partial support by the Italian Space Agency under contract ASI-INAF I/057/08/0.

\section{REFERENCES}

Daddi, E. et al. 2007, ApJ, 670, 173

Dey, A. et al. 2008, ApJ, 677, 943

Di Matteo, T., Springel, V., Hernquist, Lars. 2005, Nature, 433, 604

Fabian, A. 1999, MNRAS, 208, 39

Fiore, F. et al. 2008, ApJ, 672, 94

Fiore, F. et al. 2009, ApJ, 693, 447

Fritz, j. et al. 2006, MNRAS, 366, 767

Gruppioni, C., et al. 2008, ApJ, 684, 136

Gruppioni, C. \& Vignali, C. 2009, Spitzer Proposal ID 50476

Hopkins, P. F. et al. 2006, ApJS 163, 1

Houck, J. R. et al. 2005, ApJ, 622, 105

Lonsdale, C. et al. 2004, ApJS, 154, 54L

Magorrian, J., Tremaine, S., Richstone, D. 1998 , AJ, 115, 2285

Menci, N. et al., 2008, ApJ, 686, 219

Page, M. J. et al. 2004, ApJ, 611, 85

Polletta, M. et al. 2007, ApJ, 663, 81

Polletta, M. et al. 2008, ApJ, 675, 960

Pozzi, F. et al. 2007, A\&A, 468, 603

Tozzi, P. et al. 2006, A\&A, 451, 457

Vignali, C. et al. 2009, MNRAS, 395, 2189
Weedman, D. et al. 2006, 653, 101 Yan, L. et al. 2007, ApJ, 658, 778 